\title{
Dosage de métaux par ICP-MS dans différents milieux biologiques**
}

\section{Metals determination in biological fluids by $I C P-M S^{* *}$}

\section{Laurence LABAT*, Betty DEHON, Christine DHORNE, Michel LHERMITTE}

Laboratoire de Biochimie et de Biologie Moléculaire, CHRU de Lille, Hôpital Calmette, Avenue du Professeur J. Leclercq - 59037 LILLE CEDEX

*Auteur à qui adresser la correspondance : Laurence LABAT, Laboratoire de Biochimie et de Biologie Moléculaire, CHRU de Lille, Hôpital Calmette, Avenue du Professeur J. Leclercq - 59037 LILLE CEDEX Tél : 0320444969 - Fax : 0320444729 - E-mail : 1.labat@chru-lille.fr

** Ce travail a été présenté en partie au $40^{\text {¿me }}$ congrès de The International Association of Forensic Toxicologists (TIAFT, Paris 2002), 26-30 août 2002 PARIS - FRANCE

(Reçu le 15 octobre 2003 ; accepté le 12 décembre 2003)

\section{RÉSUMÉ}

Le plasma à couplage inductif relié à la spectrométrie de masse (Inductively Coupled Plasma-Mass Spectrometry ou ICP-MS) associé à une préparation rapide de l'échantillon permet le dosage de nombreux métaux dans les milieux biologiques. Les dosages de plomb dans le sang total, de sélénium, de cadmium et de manganèse dans le sérum, et de plomb, de cadmium, de manganèse et de nickel dans l'urine sont réalisés en ICP-MS (Agilent 7500a). Tous les prélèvements sont dilués dans une solution acide contenant de l'acide nitrique à $1 \%$, du triton $X$ et du butanol- 1 en pourcentages variés. Pour chaque élément étudié, les courbes de calibration permettent de décrire des relations linéaires $\left(r^{2}\right)$ 0,99). Les répétabilités et les fidélités intermédiaires sont toutes satisfaisantes avec des coefficients de variation compris entre 1,09\% pour le plomb dans les urines et $4,22 \%$

\section{SUMMARY}

Inductively Coupled Plasma-Mass Spectrometry (ICP-MS) with fast sample preparative procedure allows determination of numerous metals in biological fluids. Determinations of lead in whole blood, selenium, cadmium, manganese in serum and lead, cadmium, manganese and nickel in urine are described in ICP-MS (Agilent 7500a). All the samples were diluted in acidic solution with nitric acid (I\%), triton $X$ and butanol- $I$ in different proportions. For each metal, calibration curves described linear relationship $\left(r^{2}>0.99\right)$. Repeatabilities and intermediate fidelities are all satisfactory with RSD values between $1.09 \%$ (for lead in urines) and $4.22 \%$ for manganese in serum. The limits of detection are estimated to be between $0.01 \mu \mathrm{g} / \mathrm{L}$ for cadmium in urine and $0.14 \mu \mathrm{g} / 100 \mathrm{ml}$ for lead in whole blood. We described methods with good precision and they are applied to refe- 
pour le manganèse dans le sérum. Les limites de détection vont de 0,01 $\mu \mathrm{g} / \mathrm{L}$ pour le cadmium dans les urines à 0,14 $\mu \mathrm{g} / 100 \mathrm{ml}$ pour le plomb dans le sang total. Les différentes méthodes décrites sont précises et ont été appliquées avec succès à différents milieux biologiques de référence.

L'ensemble de ces résultats montre que des techniques rapides de préparation de l'échantillon comme de simples dilutions isotopiques, couplées à l'ICP-MS peuvent être utilisées en routine pour le dosage spécifique de certains métaux comme le plomb, le sélénium, le cadmium, le manganèse ou le nickel. Ce sont des méthodes précises et qui apparaissent plus sensibles que les techniques en spectrophotométrie d'absorption atomique classiquement utilisées pour ces dosages. Ces méthodes sont également tout à fait utilisables avec des dilutions d'échantillons plus importantes, dans les cas d'intoxications mettant en jeu des concentrations plus élevées. Avec de telles méthodes, l'ICP. $M S$ devient un outil performant dans les différents domaines de la toxicologie.

MOTS-CLÉS

Plasma à couplage inductif, spectrométrie de masse, métaux.

\section{Introduction}

L'évolution récente du plasma à couplage inductif relié à la spectrométrie de masse (Inductively Coupled Plasma - Mass Spectrometry ou ICP-MS) représente un apport intéressant en biologie clinique ainsi que dans le domaine de la toxicologie. Il est devenu un outil puissant pour le dosage d'un ou de plusieurs métaux dans les échantillons biologiques (1). Il apparaît ainsi comme une technique idéale en toxicologie professionnelle et environnementale pour l'analyse de certains éléments dans des populations professionnellement exposées ou dans des groupes de populations générales (2-4). Son intérêt en toxicologie clinique ou médicolégale dans des cas d'intoxications aiguës est également très important.

Actuellement, l'ICP-MS est l'une des techniques analytiques les plus sensibles existantes pour le dosage individuel d'éléments à l'état de traces dans les milieux biologiques. Son intérêt est également lié à la possibilité du dosage simultané de l'ensemble ou d'une partie de ces éléments (5-7). Il combine ainsi les avantages d'un dosage multi-élémentaire, déjà connus par ICPAES (ICP couplée à une spectrométrie d'émission) (8) et les performances en terme de sensibilité de la spectrométrie d'absorption atomique en four graphite (SAA-GF). L'ICP-MS permet même d'atteindre des niveaux de sensibilité jamais égalés par la SAA-GF. A côté de l'ICP-AES qui peut être utilisé pour le dosage d'éléments présents à des concentrations relativement élevées (alcalins, alcalino-terreux, fer, zinc, cuivre par exemple), l'ICP-MS semble la technique la plus adaptée au dosage d'une grande partie des autres éléments.

L'ICP-MS est la combinaison d'un plasma qui génère rence materials with satisfactory.

All the results suggest that a sample preparative procedure like isotopic dilution coupled with ICP-MS can be used for routine determination of metals as lead, selenium, cadmium, manganese or nickel in different matrices. These methods are precise and seem to be more sensible than absorption atomic spectrometric ones, classically used. With higher dilutions, they can be used for severe poisoning. With these methods, ICP-MS become a performing tool in different fields of toxicology.

\section{KEY-WORDS}

ICP-MS, metals.

des ions simplement chargés à partir des éléments d'un échantillon et d'un spectromètre de masse de type quadripolaire qui sépare les ions selon leur rapport masse/charge. Il utilise classiquement un plasma d'argon, obtenu par atomisation et ionisation de l'élément à une température de l'ordre de $7000 \mathrm{~K}$. A cette température, plus de soixante dix éléments sont ionisés à plus de $90 \%$. Un certain nombre d'éléments non métalliques ayant une énergie première d'ionisation élevée (phospore, soufre, arsenic, sélénium) sont également ionisés, mais en plus faibles proportions (entre 10 et $40 \%$ ) (3).

De nombreuses méthodes de dosage d'éléments-traces en ICP-MS dans des milieux biologiques variés (sang total, urine, sérum, cheveux...) sont décrites dans la littérature (1,9-11). Les avantages de ces méthodes par ICP-MS sont des temps d'analyse relativement courts, des limites de détection basses et des précisions élevées. Les méthodes de préparation d'échantillons se veulent également de plus en plus courtes et simplifiées pour une utilisation en routine de ces méthodes de dosage.

L'intérêt du dosage de ces différents éléments est décrit depuis longtemps notamment dans le domaine de la toxicologie professionnelle (12). Le tableau I décrit les principales sources d'exposition et les origines de ces différents éléments. Nous proposons plusieurs méthodes de dosage de métaux à l'état de traces dans divers milieux biologiques : le dosage du plomb dans le sang total, du sélénium, du cadmium et du manganèse dans le sérum, et du plomb, du cadmium, du manganèse et du nickel dans l'urine. Les différentes méthodes de dosage décrites restent spécifiques à chaque élément, mais sont tout de même similaires. 
Tableau I : Principales caractéristiques des métaux étudiés (d'après (14)).

\begin{tabular}{|c|c|c|c|}
\hline Élément & $\begin{array}{l}\text { Origines et sources } \\
\text { d'exposition }\end{array}$ & $\begin{array}{l}\text { Valeurs population } \\
\text { générale }\end{array}$ & $\begin{array}{c}\text { Valeurs guides de } \\
\text { l'exposition } \\
\text { professionnelle utilisables } \\
\text { en France } \\
\end{array}$ \\
\hline $\mathrm{Cd}$ & $\begin{array}{l}\text { Alimentation, tabac } \\
\text { se fixe à l'hémoglobine et } \\
\text { à la métallothionéine } \\
\text { Toxique industriel }\end{array}$ & $\begin{array}{l}\text { Sang total : } \\
<5 \mu \mathrm{g} / \mathrm{l} \text { fumeurs } \\
<1 \mu \mathrm{g} / \mathrm{l} \text { non fumeurs } \\
\text { Urine }:<2 \mu \mathrm{g} / \mathrm{g} \text { créatinine }\end{array}$ & Urine : $5 \mu \mathrm{g} / \mathrm{g}$ créatinine \\
\hline $\mathrm{Mn}$ & $\begin{array}{l}\text { Oligo-élément essentiel à } \\
\text { l'activité de différentes enzymes } \\
\text { (métabolisme des protéines, } \\
\text { synthèse du cholestérol...) } \\
\text { Toxique industriel }\end{array}$ & Urine $:<2 \mu \mathrm{g} / \mathrm{g}$ créatinine & \\
\hline $\mathrm{Ni}$ & Toxique industriel & Urine $:<3 \mu \mathrm{g} / \mathrm{g}$ créatinine & \\
\hline $\mathrm{Pb}$ & $\begin{array}{l}\text { Saturnisme } \\
\text { Alimentation (eaux) } \\
\text { Toxique industriel et polluant } \\
\text { de l'environnement }\end{array}$ & $\begin{array}{l}\text { Sang total }:<10 \mu \mathrm{g} / 100 \mathrm{~mL} \\
\text { Urine }:<25 \mu \mathrm{g} / \mathrm{g} \text { créatinine } \\
\text { (plomburie spontanée) }\end{array}$ & Sang total $:<40 \mu \mathrm{g} / 100 \mathrm{~mL}$ \\
\hline $\mathrm{Se}$ & $\begin{array}{l}\text { Oligo-élément essentiel } \\
\text { Alimentation } \\
\text { Toxique industriel } \\
\text { Anti cancérigène? }\end{array}$ & Plasma : 60 à $120 \mu \mathrm{g} / \mathrm{L}$ & \\
\hline
\end{tabular}

\section{Matériels et méthodes}

\section{Réactifs et solutions étalons}

Tous les réactifs utilisés sont de qualité pour analyse. Ils ont été évalués et choisis pour ne pas contribuer à de possibles contaminations des solutions de dilutions (teneur pour chaque métal garantie inférieure à $0,00001 \%)$. L'acide nitrique (65\%) a été obtenu chez Riedel de Haën (Seelz, Allemagne), le 1-butanol Rectapur chez Prolabo (Fontenay sous Bois, France) et le Triton X $100 \mathrm{chez}$ Sigma Aldrich (Steinheim, Allemagne). L'eau utilisée est de l'eau désionisée.

Des solutions étalons de séléniüm, plomb, manganèse, cadmium ou nickel à $1 \mathrm{~g} / \mathrm{L}$ ont été obtenues chez Merck (Darmstadt, Allemagne). A partir de chacune de ces solutions étalons, des solutions de travail ont été préparées (concentrations de 10 ou $100 \mu \mathrm{g} / \mathrm{L}$ ) après dilution dans les solutions acides de compositions variées (détaillées dans le tableau II). Chacune de ces solutions de travail est utilisée pour la calibration.

Différents contrôles ont été utilisés. Il s'agit des contrôles Utak Labs (Valencia, USA) dans le sang total pour le cadmium, dans le sérum pour le sélénium et le manganèse et dans l'urine pour le plomb, le cadmium, le manganèse et le nickel, et de contrôles Seronorm (Fumouze, Levallois-Perret, France) pour le sélénium dans le sérum et le plomb dans le sang total.

\section{Préparation de l'échantillon}

Les contrôles et les échantillons à analyser sont dilués $(1: 9$ ou $1: 49, v / v)$ dans des solutions acides dont la composition varie selon l'élément à doser (tableaux II et III).

Tableau II : Composition des solutions utilisées pour le dosage des métaux en ICP-MS.

\begin{tabular}{|l|c|c|c|}
\hline Élément à doser & \multicolumn{3}{|c|}{ Solution de dilution } \\
& triton X 100 (\%) & butanol-1 (\%) & acide nitrique (\%) \\
\hline Cd (urine) & 0,1 & 0,2 & 1 \\
Cd (sang total) & 0,1 & 0,5 & 1 \\
Mn (sérum) & 0,1 & 1 & 1 \\
Mn (urine) & 0,1 & 1 & 1 \\
Ni (urine) & 0,1 & 0,2 & 1 \\
Pb (sang total) & 0,1 & 0,2 & 1 \\
Pb (urine) & 0,1 & 0,5 & 1 \\
Se (plasma) (9) & 0,1 & 0,8 & 1 \\
\hline
\end{tabular}


Tableau II : Linéarité des méthodes.

\begin{tabular}{|l|c|c|c|}
\hline Elément à doser & $\begin{array}{c}\text { Gamme } \\
\text { d'étalonnage }\end{array}$ & $\begin{array}{c}\text { Facteur de } \\
\text { dilution des } \\
\text { échantillons }\end{array}$ & $\begin{array}{c}\text { Coefficients de } \\
\text { régression }\end{array}$ \\
\hline Cd (urine) & $0,25-2 \mu \mathrm{g} / \mathrm{L}$ & $1 / 10$ & 0,999 \\
$\mathrm{Cd}$ (sang total) & $0,25-2 \mu \mathrm{g} / \mathrm{L}$ & $1 / 10$ & 0,999 \\
Mn (sérum) & $0,25-2 \mu \mathrm{g} / \mathrm{L}$ & $1 / 10$ & 0,999 \\
$\mathrm{Mn}$ (urine) & $0,25-2 \mu \mathrm{g} / \mathrm{L}$ & $1 / 10$ & 0,999 \\
$\mathrm{Ni}$ (urine) & $2-10 \mu \mathrm{g} / \mathrm{L}$ & $1 / 10$ & 0,999 \\
$\mathrm{~Pb}$ (sang total) & $0,5-7 \mu \mathrm{g} / 100 \mathrm{~mL}$ & $1 / 10$ & 0,999 \\
$\mathrm{~Pb}$ (urine) & $5-20 \mu \mathrm{g} / \mathrm{L}$ & $1 / 50$ & 0,999 \\
$\mathrm{Se}$ (sérum) & $1-40 \mu \mathrm{g} / \mathrm{L}$ & $1 / 10$ & 0,999 \\
\hline
\end{tabular}

\section{Appareillage}

Les analyses sont réalisées sur un plasma à couplage inductif relié à la spectrométrie de masse, (ICP-MS Agilent 7500a, Agilent technologies, Evry, France). L'ICP-MS est pilotée par un système informatique Chemstation Agilent 7500a. Les principales conditions opératoires de l'ICP-MS sont résumées dans le tableau IV. Tous ces paramètres ont été ajustés afin d'obtenir un signal satisfaisant pour l'ensemble des éléments à analyser. Les échantillons dilués sont introduits directement dans le plasma par un nébulisateur de type babington. Avant et après chaque injection, les tubulures du système sont conditionnées par rinçage avec de l'acide nitrique à $1 \%$.

Tableau IV : Paramètres instrumentaux de l'ICP-MS.

\begin{tabular}{|l|l|}
\hline Puissance de la torche & $1300 \mathrm{~W}$ \\
Débit de l'argon pour l'alimentation du plasma & $16 \mathrm{~L} / \mathrm{min}$ \\
Débit de l'argon auxiliaire & $0 \mathrm{~L} / \mathrm{min}$ \\
Débit de l'argon du nébuliseur & $1,23 \mathrm{l} / \mathrm{min}$ \\
Masse spécifique utilisée pour chacun des éléments (m/z) & $\mathrm{Cd} 111$ \\
& $\mathrm{Mn} \mathrm{55}$ \\
& $\mathrm{Ni} 60$ \\
& $\mathrm{~Pb} \mathrm{208}$ \\
& $\mathrm{Se} 82$ \\
\hline
\end{tabular}

\section{Résultats}

Le tableau IV montre pour chacun des éléments, l'isotope choisi permettant la détection et le dosage. Tous les paramètres influençant le dosage ont été étudiés pour chaque élément. L'importance de la présence et du pourcentage en triton X 100 et en butanol-1 dans la solution acide ont été examinées. Différentes concentrations ont été testées entre 0 et $1 \%$ pour le butanol-1 et 0 et $0,2 \%$ pour le triton $X 100$. Il apparait comme impossible d'envisager le dosage des éléments dans les différents milieux biologiques sans la présence de ces deux composés organiques.

Aucune des méthodes de dosage décrites n'a nécessité l'utilisation d'un étalon interne. Des relations linéaires ont été observées entre les réponses obtenues (en nombre de coups par seconde) et les concentrations des différents éléments. Leurs coefficients de régression sont supérieurs à 0,999 pour chaque élément (voir tableau III). Les limites de détection (Ldd) et de quantification (Ldq) ont été estimées pour chaque élément à partir de la mesure du bruit de fond d'une solution correspondant à la solution acide servant à la dilution des échantillons. Les Ldd et Ldq sont définies respectivement par la valeur moyenne de ce bruit de fond, mesurée sur 30 échantillons à laquelle on ajoute respectivement 3 et 10 écarts type. L'ensemble de ces valeurs est reporté dans le tableau V. La répétabilité et la fidélité intermédiaire ont été déterminées pour une concentration pour chaque élément (voir tableau $\mathrm{V}$ ). Les résultats sont tout à fait satisfaisants avec des coefficents de variation exprimés en pourcentage compris entre $1,09 \%$ et $4,22 \%$.

La précision de chaque méthode a été mesurée par la mesure des coefficients de variation pour six mesures de chaque élément dans des milieux de référence correspondant à des contrôles de qualités externe. Les résultats sont regroupés tableau VI.

\section{Discussion}

Nous proposons différentes méthodes de dosage en ICP-MS du plomb dans le sang total, du sélénium, du cadmium et du manganèse dans le sérum, et du plomb, du cadmium, du manganèse et du nickel dans l'urine. L'ensemble des résultats montrent qu'il existe une excellente corrélation entre les réponses et les concentrations en métaux dans les trois types de milieux biologiques. Pour chacun d'entre eux, les gammes réalisées en solution aqueuse et dans la matrice étaient superposables, montrant l'absence d'un effet de matrice (données non montrées). Toutes les gammes ont ainsi été réalisées simplement dans de l'eau en milieu acide. Les valeurs des limites de quantification sont toujours plus faibles que celles des valeurs de la population générale (voir tableau I). L'utilisation de ces méthodes de dosage pour des personnes exposées en milieu professionnel est donc tout à fait adaptée.

Parmi les nombreuses méthodes décrites dans la littérature pour les différents éléments étudiés, l'ICP-MS présente l'avantage d'être la plus sensible. Par exemple, l'ICP-AES ne permet le dosage du sélénium qu'avec une limite de détection 20 fois plus importante que 
Tableau V : Paramètres de validation des méthodes de dosage des différents métaux en ICP-MS.

\begin{tabular}{|l|c|c|c|c|}
\hline Élément à doser & Ldd & Ldq & $\begin{array}{c}\text { Répétabilité (\%) } \\
(\mathbf{n = 6})\end{array}$ & $\begin{array}{c}\text { Fidélité intermédiaire (\%) } \\
(\mathbf{n}=6)\end{array}$ \\
\hline Cd (urine) & $0,01 \mu \mathrm{g} / \mathrm{L}$ & $0,03 \mu \mathrm{g} / \mathrm{L}$ & 2,19 & 2,49 \\
Cd (sang total) & $0,02 \mu \mathrm{g} / \mathrm{L}$ & $0,03 \mu \mathrm{g} / \mathrm{L}$ & 1,24 & 2,11 \\
Mn (sérum) & $0,06 \mu \mathrm{g} / \mathrm{L}$ & $0,16 \mu \mathrm{g} / \mathrm{L}$ & 3,49 & 4,22 \\
Mn (urine) & $0,06 \mu \mathrm{g} / \mathrm{L}$ & $0,16 \mu \mathrm{g} / \mathrm{L}$ & 3,68 & 3,77 \\
$\mathrm{Ni}$ (urine) & $0,16 \mu \mathrm{g} / \mathrm{L}$ & $0,45 \mu \mathrm{g} / \mathrm{L}$ & 1,94 & 3,29 \\
$\mathrm{~Pb}$ (sang total) & $0,13 \mu \mathrm{g} / 100 \mathrm{~mL}$ & $0,14 \mu \mathrm{g} / 100 \mathrm{~mL}$ & 2,39 & 2,64 \\
$\mathrm{~Pb}$ (urine) & $0,22 \mu \mathrm{g} / \mathrm{L}$ & $0,30 \mu \mathrm{g} / \mathrm{L}$ & 1,09 & 2,57 \\
$\mathrm{Se}$ (sérum) & $0,31 \mu \mathrm{g} / \mathrm{L}$ & $1,00 \mu \mathrm{g} / \mathrm{L}$ & 2,00 & 3,22 \\
\hline
\end{tabular}

Tableau VI : Précision des méthodes de dosage en ICP-MS.

\begin{tabular}{|l|c|c|c|c|}
\hline Élément à doser & Contrôles & Valeurs certifiées & Valeurs mesurées & $\begin{array}{c}\text { CV (\%) } \\
\text { (n=6) }\end{array}$ \\
\hline Cd (urine) & UTAK Urine & $0,52 \mu \mathrm{g} / \mathrm{L}$ & $0,50 \mu \mathrm{g} / \mathrm{L}$ & 4,20 \\
& UTAK Urine & $5,26 \mu \mathrm{g} / \mathrm{L}$ & $5,33 \mu \mathrm{g} / \mathrm{L}$ & 2,60 \\
Cd (sang total) & UTAK Whole blood & $0,70 \mu \mathrm{g} / \mathrm{L}$ & $0,68 \mu \mathrm{g} / \mathrm{L}$ & 4,89 \\
Mn (sérum) & UTAK Whole blood & $10,7 \mu \mathrm{g} / \mathrm{L}$ & $10,3 \mu \mathrm{g} / \mathrm{L}$ & 2,11 \\
& UTAK Serum & $0,80 \mu \mathrm{g} / \mathrm{L}$ & $0,86 \mu \mathrm{g} / \mathrm{L}$ & 5,72 \\
Mn (urine) & UTAK Serum & $2,40 \mu \mathrm{g} / \mathrm{L}$ & $2,51 \mu \mathrm{g} / \mathrm{L}$ & 7,87 \\
& UTAK Urine & $5,65 \mu \mathrm{g} / \mathrm{L}$ & $5,77 \mu \mathrm{g} / \mathrm{L}$ & 3,03 \\
Ni (urine) & UTAK Urine & $2,75 \mu \mathrm{g} / \mathrm{L}$ & $2,86 \mu \mathrm{g} / \mathrm{L}$ & 5,63 \\
& UTAK Urine & $2,50 \mu \mathrm{g} / \mathrm{L}$ & $2,42 \mu \mathrm{g} / \mathrm{L}$ & 1,94 \\
Pb (sang total) & UTAK Urine & $40 \mu \mathrm{g} / \mathrm{L}$ & $39 \mu \mathrm{g} / \mathrm{L}$ & 0,95 \\
& Seronorm Whole Blood & $3,3 \mu \mathrm{g} / 100 \mathrm{~mL}$ & $3,4 \mu \mathrm{g} / 100 \mathrm{~mL}$ & 3,52 \\
Pb (urine) & Seronorm Whole Blood & $64,1 \mu \mathrm{g} / 100 \mathrm{~mL}$ & $64,6 \mu \mathrm{g} / 100 \mathrm{~mL}$ & 3,43 \\
& UTAK Urine & $<5 \mu \mathrm{g} / \mathrm{L}$ & $4,5 \mu \mathrm{g} / \mathrm{L}$ & 1,49 \\
Se (sérum) & UTAK Urine & $157 \mu \mathrm{g} / \mathrm{L}$ & $135 \mu \mathrm{g} / \mathrm{L}$ & 0,99 \\
& Seronorm Trace Elements Serum & $83 \mu \mathrm{g} / \mathrm{L}$ & $84 \mu \mathrm{g} / \mathrm{L}$ & 2,00 \\
& UTAK Serum & $304 \mu \mathrm{g} / \mathrm{L}$ & $316 \mu \mathrm{g} / \mathrm{L}$ & 1,85 \\
\hline
\end{tabular}

celle que nous décrivons (9). Comparé aux techniques d'utilisation de l'ICP-MS en mode multi élémentaire, les performances des méthodes spécifiques de dosage sont souvent améliorées en terme de sensibilité, de précision ou de reproductibilité $(5,8)$. Goullé et coll. décrivent des valeurs pour les limites de détection en général toujours plus élevées, pouvant aller jusqu'à 5 ou 20 fois plus pour le manganèse ou le sélénium respectivement dans le sérum (5).

Avec l'ICP, des problèmes d'interférences ont été décrits lors du dosage de certains éléments. On peut remédier à ce problème en utilisant des cellules dynamiques de réaction. Pour le dosage du sélénium, l'iso- tope le plus abondant, le ${ }^{80} \mathrm{Se}$ (abondance naturelle de $49,8 \%$ ) ne peut être utilisé pour une détection en spectrométrie de masse en raison de l'interférence connue avec le dimère $\mathrm{Ar}_{2}(\mathrm{~m} / \mathrm{z} 80)$. Seul le choix d'une cellule dynamique de réaction ou d'un autre isotope comme ${ }^{82}$ Se permet de s'affranchir de cette interférence (9). A notre connaissance, aucune méthode ne décrit le dosage de cet élément avec une cellule dynamique de réaction dont l'utilisation reste plus délicate.

Notre étude décrit des procédures simples et rapides de préparation de l'échantillon permettant le dosage direct des éléments traces dans différents milieux biologiques en ICP-MS. Ces méthodes de dosage présentent l'avan- 
tage de ne pas nécessiter la mise en œuvre de préparations d'échantillons plus lourdes comme par exemple l'extraction des prélèvements biologiques ou la génération d'hydrures. La dilution isotopique réalisée pour chaque élément permet de s'affranchir d'éventuelles amplifications de signal dues à la présence de carbone en proportion variable dans les différentes matrices étudiées (13). La présence de butanol-1 et donc de carbone dans l'ensemble des échantillons, semble être indispensable pour atténuer les effets de la matrice. En effet, une concentration homogène en carbone minimise les interactions entre les molécules de la matrice et les ions responsable de ces effets de matrice. Pour chaque couple élément à doser - matrice biologique, la concentration en butanol-1 a été optimisée dans ce sens.

\section{Conclusion}

Nous proposons des méthodes rapides et simples de dosage en ICP-MS de quelques éléments à l'état de trace dans des matrices différentes : le plomb dans le sang total, le sélénium, le cadmium et le manganèse dans le sérum, et le plomb, le cadmium, le manganèse et le nickel dans l'urine. Nous montrons ainsi qu'un même matériel et une préparation de l'échantillon rapide par simple dilution des prélèvements en milieu acide permet le dosage d'éléments différents à l'état de trace.

Ce sont des méthodes faciles à mettre en ouvre dans tous les laboratoires possédant une ICP-MS. A côté des performances de ce type de technologie maintenant reconnues pour l'analyse multi élémentaire, les méthodes de dosage décrites nous montrent l'intérêt de l'utilisation de l'ICP-MS pour le dosage spécifique de certains éléments à côté de techniques plus classiques comme la spectrométrie d'absorption atomique.

\section{Références}

1. Nuttall K.L., Gordon W.H., Ash K.O. Inductively coupled plasma mass spectrometry for trace element analysis in the clinical laboratory. Ann. Clin. Lab. Sci. $1995 ; 25$ : 264-71.

2. Dehon B., Nisse C., Lhermitte M., Haguenoer J.M. Métaux et médecine du travail. Ann. Toxicol. Anal. $2001 ; 13: 203-19$.

3. Apostoli P. Elements in environmental and occupational medicine. J. Chromatogr. B. $2002 ; 778 ; 63-97$.

4. White M.A. A comparison of inductively coupled plasma mass spectrometry with electrothermal atomic absorption spectrometry for the determination of trace elements in blood and urine from non occupationally exposed populations. J. Trace Elem. Med. Biol, 1999 ; 13 : 93-101.
5. Goullé J.P., Mahieu L ., Castermant J., Neveu N., Laine G., Nouveau M.P., Gehanne R., Bouige D., Lacroix C. Validation d'une technique de dosage multi-élémentaire des métaux par ICP-MS dans les milieux biologiques. Ann. Toxicol. Anal. 2003 ; XV (4) : 271-80.

6. Forrer R., Gautschi K., Lutz H. Simultaneous measurement of the trace elements $\mathrm{Al}, \mathrm{As}, \mathrm{B}, \mathrm{Be}, \mathrm{Cd}, \mathrm{Co}, \mathrm{Cu}, \mathrm{Fe}$, $\mathrm{Li}, \mathrm{Mn}, \mathrm{Mo}, \mathrm{Ni}, \mathrm{Rb}, \mathrm{Se}, \mathrm{Sr}$, and $\mathrm{Zn}$ in human serum and their reference ranges by ICP-MS. Biol. Trace Elem. Res. $2001 ; 80: 77-93$.

7. Krachler M., Irgolic K.J. The potential of inductively coupled plasma mass spectrometry (ICP-MS) for the simultaneous determination of trace elements in whole blood, plasma and serum. J. Trace Elem. Med. Biol. 1999 ; 13 : 157-69.

8. Rahil-Khazen R., Henriksen H., Bolann B.J., Ulvik R.J. Validation of inductively coupled plasma atomic emission spectrometry technique (ICP-AES) for multi-element analysis of trace elements in human serum. Scan. J. Clin. Lab. Invest. $2000 ; 60: 677-86$.

9. Labat L., Dehon B., Lhermitte M. Rapid and simple determination of selenium in blood serum by inductively coupled plasma-mass spectrometry (ICP-MS). Anal. Bioanal. Chem. 2003 ; 376 : 270-3.

10. Labat L., Dehon B., Dhorne C., Lhermitte M. Usefulness of ICP-MS for the determination of trace metals in various matrices. Ann. Toxicol. Anal. 2002 ; XIV (3) : 291.

11. Schutz A., Bergdahl I., Ekholm A., Skerfving S. Measurement by ICP-MS of lead in plasma and whole blood of lead workers and controls. Occup. Environ. Med. 1996 ; 53 : 736-40.

12. Lauwerys R. Toxicologie industrielle et intoxications professionnelles. Paris, Masson (4 ${ }^{\text {eme }}$ édition), 2003, 96/pp.

13. Hsiung C.S., Andrade J.D., Costa R., Ash K.O. Minimizing interferences in the quantitative multielement analysis of trace elements in biological fluids by inductively coupled plasma mass spectrometry. Clin. Chem. 1997 ; 43 : 2303-11.

14. BIOTOX. Inventaire des laboratoires effectuant des dosages biologiques de toxiques industriels. INRS, 2002. 